\title{
Dosage, Efficacy and Safety of Cannabidiol Administration in Adults: A Systematic Review of Human Trials
}

\author{
Christian Larsen ${ }^{\mathrm{a}, \mathrm{b}}$, Jorida Shahinas ${ }^{\mathrm{a}}$
}

\begin{abstract}
Considering data from in vitro and in vivo studies, cannabidiol (CBD) seems to be a promising candidate for the treatment of both somatic and psychiatric disorders. The aim of this review was to collect dose(s), dosage schemes, efficacy and safety reports of CBD use in adults from clinical studies. A systematic search was performed in PubMed, Embase and Cochrane library for articles published in English between January 1, 2000 and October 25, 2019. The search terms used were related to cannabis and CBD in adults. We identified 25 studies (927 patients; 538 men and 389 women), of which 22 studies were controlled clinical trials (833 patients) and three were observational designs (94 patients) from five countries. Formulations, dose and dosage schemes varied significantly between studies. Varying effects were identified from the randomized controlled trials (RCTs), more apparent effects from non-RCTs and minor safety issues in general. From the controlled trials, we identified anxiolytic effects with acute CBD administration, and therapeutic effects for social anxiety disorder, psychotic disorder and substance use disorders. In general, studies were heterogeneous and showed substantial risks of bias. Although promising results have been identified, considerable variation in dosage schemes and route of administration were employed across studies. There was evidence to support single dose positive effect on social anxiety disorder, short medium-term effects on symptomatic improvement in schizophrenia and lack of effect in the short mediumterm on cognitive functioning in psychotic disorders. Overall, the administration was well tolerated with mild side effects.
\end{abstract}

Keywords: Cannabidiol; Dose; Safety; Efficacy; Cannabis

\section{Introduction}

Cannabis sativa L. has a long tradition of medical use. However, its clinical use has been limited due to the effects on the central nervous system and the possibility of drug abuse and addiction. The plant exudes a resin containing a mix of cannab-

Manuscript submitted January 15, 2020, accepted February 5, 2020

aNordic Cannabis Research Institute, Copenhagen, Denmark

${ }^{b}$ Corresponding Author: Christian Larsen, Nordic Cannabis Research Institute, Copenhagen, Denmark. Email: cl@ncrinstitute.com

doi: https://doi.org/10.14740/jocmr4090 inoids with two principal components, $\Delta$ 9-tetrahydrocannbinol (THC) and cannabidiol (CBD). The structure and configuration of CBD was discovered in the 60s and has gained particular attention due to the lack of psychotropic activity. Because of its excellent tolerability in humans, the lack of psychoactive action and the low abuse potential, it seems an ideal candidate for use in a clinical context [1].

In addition to its good safety profile and the lack of psychoactive effects, CBD presents also a wide range of therapeutic effects [2]. Possibly for these reasons, CBD is currently one of the most studied cannabinoids [3]. Several experimental in vitro and in vivo studies have shown that CBD has a broad range of therapeutic applications, displaying anti-inflammatory and immunomodulatory properties [4], anti-psychotic [5], analgesic [6] and anti-epileptic [7] effects, among others. Compared to $\triangle 9$-THC, CBD shows low affinity for cannabinoid receptor type $1\left(\mathrm{CB}_{1}\right)$ and type $2\left(\mathrm{CB}_{2}\right)[8] . \mathrm{CB}_{1}$ receptors are mainly found in the terminals of central and peripheral neurons and $\mathrm{CB}_{2}$ receptors mainly in immune cells [9]. Several in vitro studies have shown that $\mathrm{CBD}$, at low concentrations, has weak $\mathrm{CB}_{1}$ and $\mathrm{CB}_{2}$ antagonistic effect [10]. It has also been reported that it behaves as a negative allosteric modulator of $\mathrm{CB}_{1}$, meaning that $\mathrm{CBD}$ does not activate the receptor directly but alter the potency and efficacy of orthosteric ligands of this receptor: $\triangle 9$-THC and 2-arachidonoylglycerol (2-AG) [11]. These preliminary results need further validation, but may explain the ability of CBD to antagonize some of the effects of $\triangle 9$-THC reported in in vitro, in vivo and clinical human studies [12]. It has also been suggested that the role of CBD as an allosteric modulator of $\mathrm{CB}_{1}$ can explain its therapeutic role in the treatment of central and peripheral nervous system disorders [2]. CBD has also shown to have a strong inhibition effect of neutrophil chemotaxis and proliferation. In addition, it may induce stimulation of arachidonic acid release, reducing prostaglandin E2 (PGE2), and nitric oxide (NO) production. Furthermore, $\mathrm{CBD}$ reduces the expression of specific interleukins (IL-12 while increasing that of IL-10) by macrophages, and decreases the production and release of pro-inflammatory cytokines, such as IL-1, IL-6 and interferon gamma (IFN $\gamma$ ) from lipopolysaccharide (LPS)-activated microglial cells [13]. The role of $\mathrm{CBD}$ as an inverse agonist of $\mathrm{CB}_{2}$ receptor may explain its known anti-inflammatory effects but this needs further investigation. There is also evidence of an antagonistic effect of $\mathrm{CBD}$ at the novel cannabinoid receptor $\mathrm{G}$ protein-coupled receptor 55 (GPR55), emerging from in vitro and in vivo studies. GPR55 has a role in bone physiology via regulating osteoclast function, formation and ultimately bone mass. CBD may affect 
the endocannabinoid system also indirectly, for example CBD can affect the endocannabinoid tone by increasing availability of anandamide; one possible mechanism is by inhibition of fatty acid amide hydrolase (FAAH), the enzyme that hydrolyzes the endocannabinoid anandamide [14].

However, not all physiological effects of CBD are mediated by cannabinoid receptors. Indeed CBD has numerous targets outside the endocannabinoid system and the cannabinoid receptor independent action is the subject of recent pharmacological studies on CBD [2]. Some of these physiological effects such as anti-inflammatory and immunosuppressive effect are mediated by more than one target [8]. The anti-inflammatory immunosuppressive effects are possibly mediated by activation of adenosine receptors, $\mathrm{A}_{1 \mathrm{~A}}$ and $\mathrm{A}_{2 \mathrm{~A}}$ and strychninesensitive $\alpha 1$ and $\alpha 1 \beta$ glycine receptors and the inhibition of the equilibrative nucleoside transporter [8]. The activity of CBD at one defined target may also elicit different physiological effects. For example, anti-inflammatory action and suppression of neuropathic pain are mediated by the same glycine receptors or anxiolytic, panicolytic and anti-depressant effects via serotonin $5 \mathrm{HT}_{1 \mathrm{~A}}$ receptor sub-type [8]. Pisano et al (2017) showed an in-depth review of the molecular pharmacology of CBD [2]. Despite the great number of studies published on molecular pharmacology of $\mathrm{CBD}$, the exact pharmacological action of CBD remains not fully characterized and ongoing efforts are directed toward fully elucidating these mechanisms [3].

The clinical studies on the effects of CBD date back to the 80 s but recently the number of studies and registered trials evaluating the effectiveness of this compound has risen exponentially. Currently, CBD is commercially available in different formulations and used for several health conditions but could be indicated for several diseases or disorders in addition to or in replacement of medical marijuana or other medical therapy. However, it remains unclear in which form and dose CBD should be administered to assess safety and efficacy across indications. This might further be complected by the nature of altered dose depending on indication of intervention. To highlight administration and dosage for known indications (e.g. diseases and disorders), this systematic review reports the current evidence, literature and experiences of how and in which dose CBD could be administered as well as efficacy and safety reports.

\section{Methods}

\section{Study eligibility criteria}

One author (CL) systematically searched PubMed, EMBASE and the Cochrane Library for published studies in English. The search was last updated on November 5, 2019. We included human studies of reporting more than 10 adult patients. We restricted our search to studies published after the year of 2000 with no demographic limitation. We included studies with single treatment of $\mathrm{CBD}$, e.g. studies with other cannabinoid add-on therapy or adjuvant regimes were excluded. Studies must report dosage and dosage schemes to be included. There was no limitation regarding study design. Studies on pediatric populations were excluded. Only studies published in English, from 2000 to the date last databases were accessed. Full-text articles were interdependently assessed by two reviewers and eventual disagreement was resolved through consensus.

PubMed, Embase and Cochrane database were searched with all combinations of CBD and dosage in human studies. Reference lists of included studies were evaluated, and if needed, authors were contacted for further information.

\section{Data collection and critical appraisal}

We extracted the following data: condition and symptoms, year of publication, country, study population, number of patients, age, gender, weight, outcome measures, effect of treatment, side effects, dosage, administration form (pills, smoking, oil, etc.), length of treatment, doses, product/brand, isolated CBD or full spectrum and funding/sponsors.

The methodological quality of randomized controlled trials (RCTs) was assessed using the Cochrane risk of bias tool [15]. If all domains were judged as being at low risk of bias, the study was considered at low risk of bias. If at least one domain was rated high, the study was considered at high risk of bias. In situations that differ from those described above, the study was considered at unclear rick of bias. Data extraction and risk of bias was assessed by two independent reviewers and disagreements were solved by a third reviewer or with consensus.

\section{Synthesis}

Clinical heterogeneity was assessed by grouping studies by indication and outcome (including disease-specific outcomes and if specified adverse events (AEs)) and scheme of drug administration. Data were too heterogeneous to be pooled, so we used a narrative synthesis.

To rate the overall quality of evidence for risk of bias, the Grading of Recommendations Assessment, Development and Evaluation (GRADE) was used for each outcome of interest across all domains: methodological limitations of the studies, indirectness, imprecision, inconsistency and publication bias [16].

\section{Results}

\section{Study selection and characteristics}

We identified 362 studies of which 85 were assessed for fulltext eligibility (Fig. 1). Finally, 25 studies were included: 22 controlled clinical trials and three non-controlled (single arm) trials [10-34]. Among controlled clinical trials, two were nonRCTs and 20 RCTs (14 individually randomized parallel group trial, five individually randomized cross-over trials and one cluster randomized cross-over trial). Studies were conducted in five countries with the majority of studies being conducted in the UK. All studies were reported in full length journal ar- 


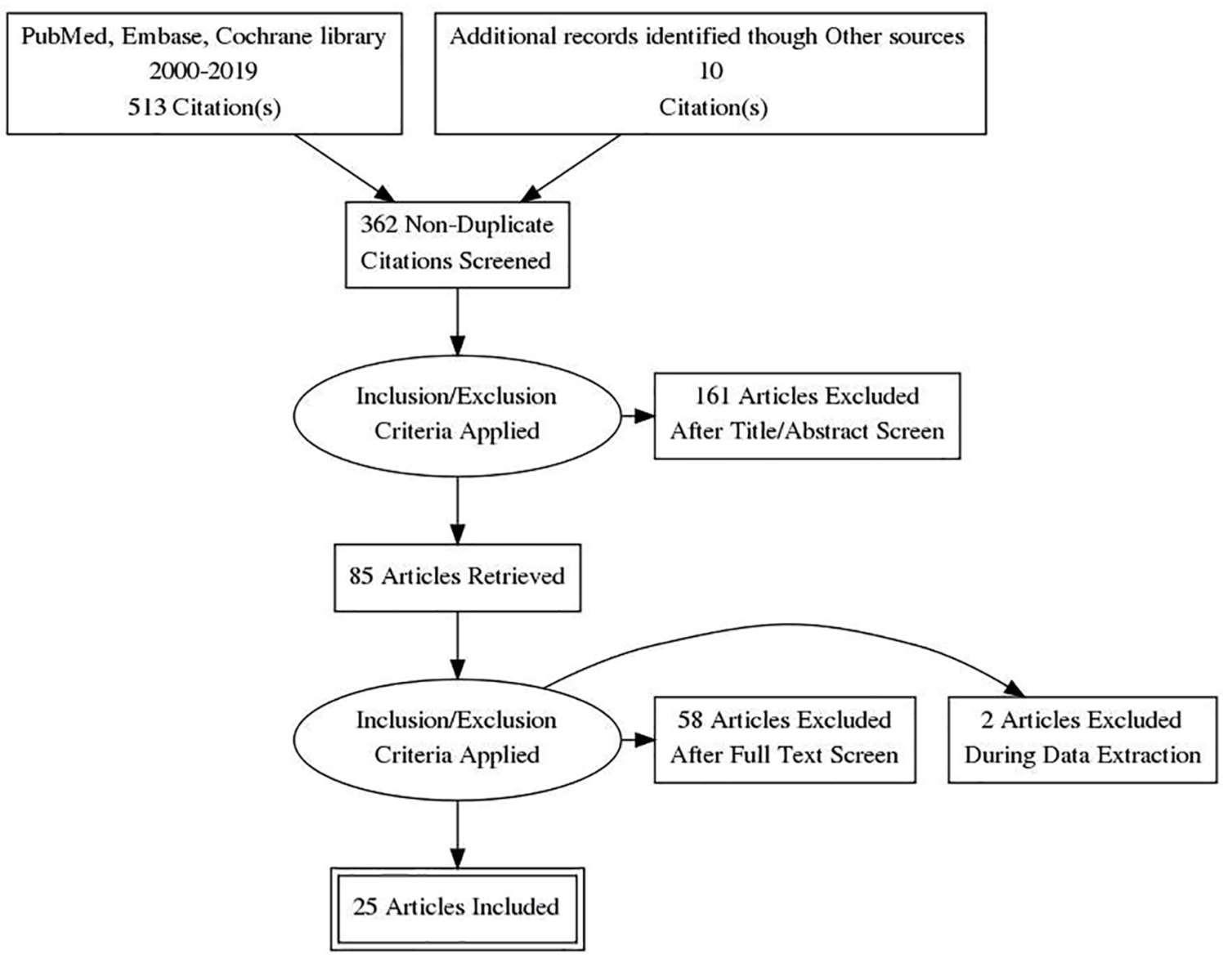

Figure 1. PRISMA flow diagram of literature search and selection process. PRISMA: Preferred Reporting Items for Systematic Reviews and Meta-Analyses.

ticles. The administration of CBD varied from single doses to chronic administration, up to 48 weeks. The pure form of CBD was used in the majority of the studies except for two studies using seed CBD oil and CBD-rich botanical extracts. The most common form of drug administration was in the form of oral capsules, and other forms were vaporization and sublingual oil. The most common comparator was placebo only one study used an active-control group. Results for controlled clinical trials and non-controlled studies are presented in Table 1 [1737] and Table 2 [38-40], providing a summary of the included studies and their principal findings.

\section{The risk of bias within studies}

Among the randomized trials, three were judged at low risk of bias, eight were judged at high risk of bias and nine at uncertain risk. Major potential sources of bias were frequent in the following domains: randomization process and selection of the reported results. Most studies reported were randomized and double-blinded but few reported methods of randomization or participants and outcome assessor blinding. Selective outcome reporting was also a potential source of bias, since some studies did not report data for all outcomes specified in the methods section or trial register or changed the primary outcome from what was specified in the trial register.

Most studies reported outcomes differently and even in cases where the same outcome was reported it was measured differently. Outcome level assessment was performed only for anxiety in social anxiety disorder (SAD) and psychotic symptoms and cognitive function in schizophrenia patients following recommendations applying GRADE approach in narrative synthesis [9]. GRADE rating is presented in Table 3. Two studies assessed anxiety in treatment-naive SAD populations, and three assessed cognitive function and positive and negative symptoms in schizophrenia patients.

\section{Anxiety disorder}

Anxiety was assessed in 11 studies (358 participants) [17-27]. Anxiety was the main outcome in eight studies, while possible anxiolytic effects were evaluated in the remaining three studies, through indirect measures such as emotional processing, reactivity to negative stimuli, etc. Among the studies providing data on anxiety, there were only two restricted analyses to patients with anxiety disorders and another to non-clinic paranoia patients. Nine studies were randomized clinical trials: one of 


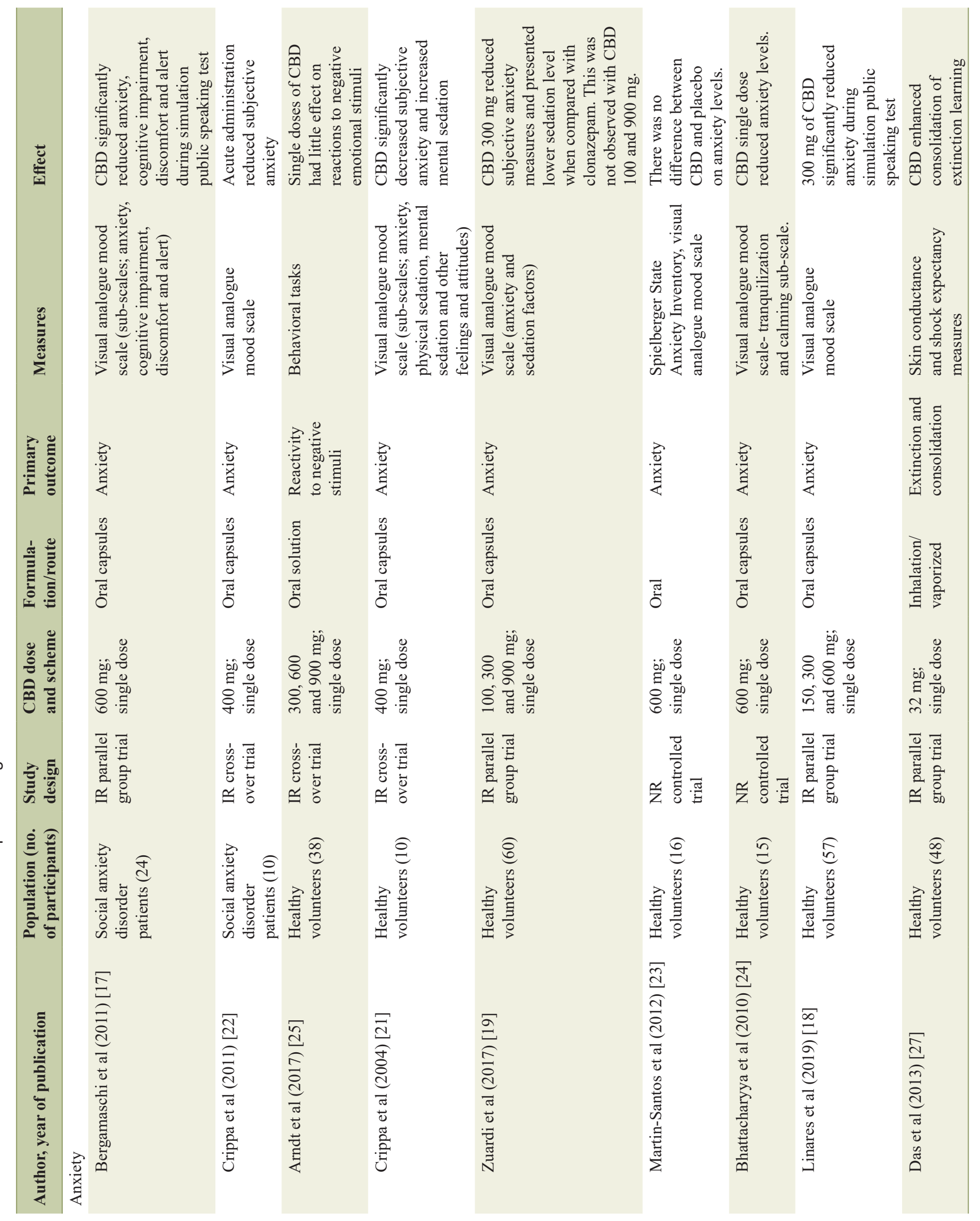




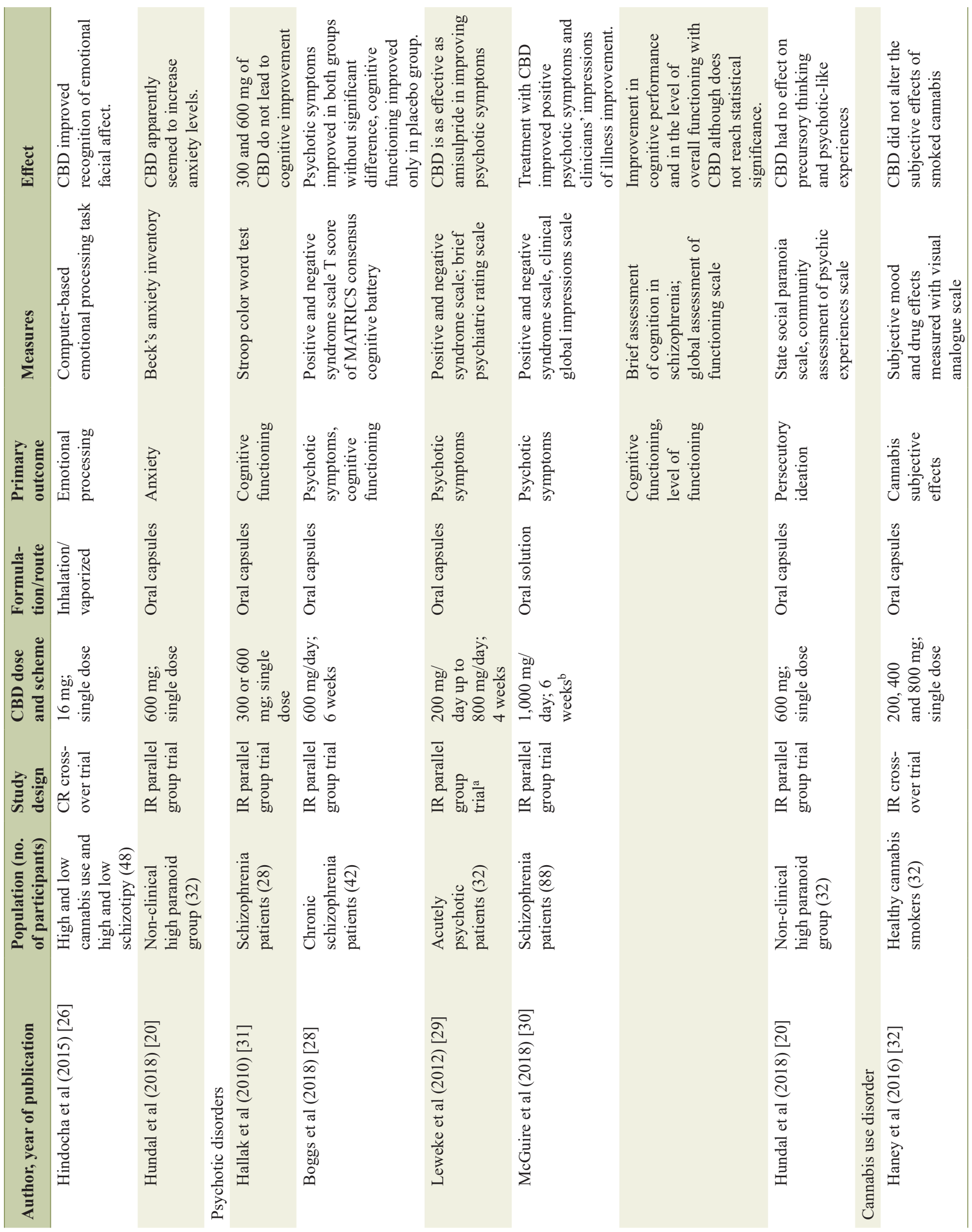




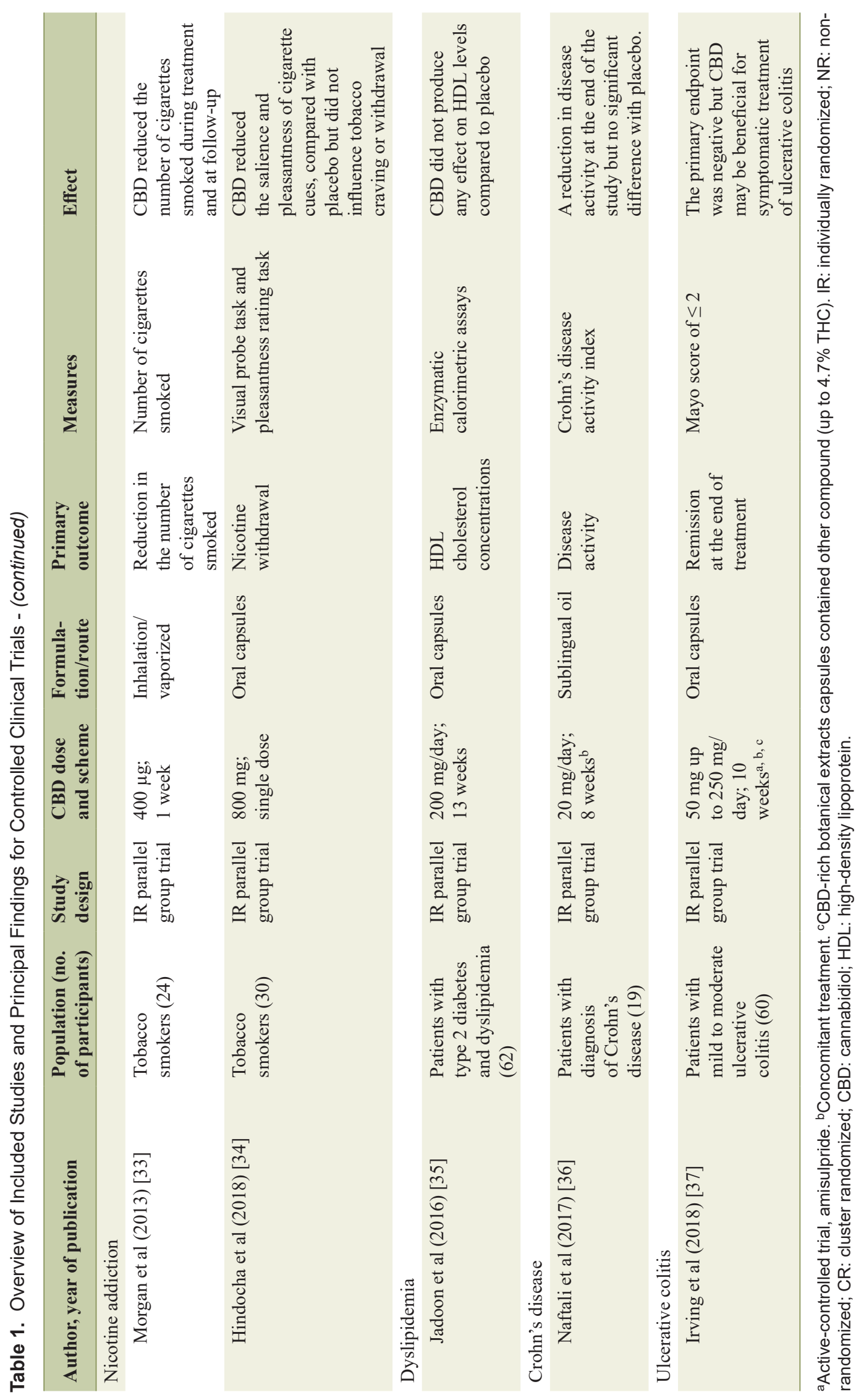




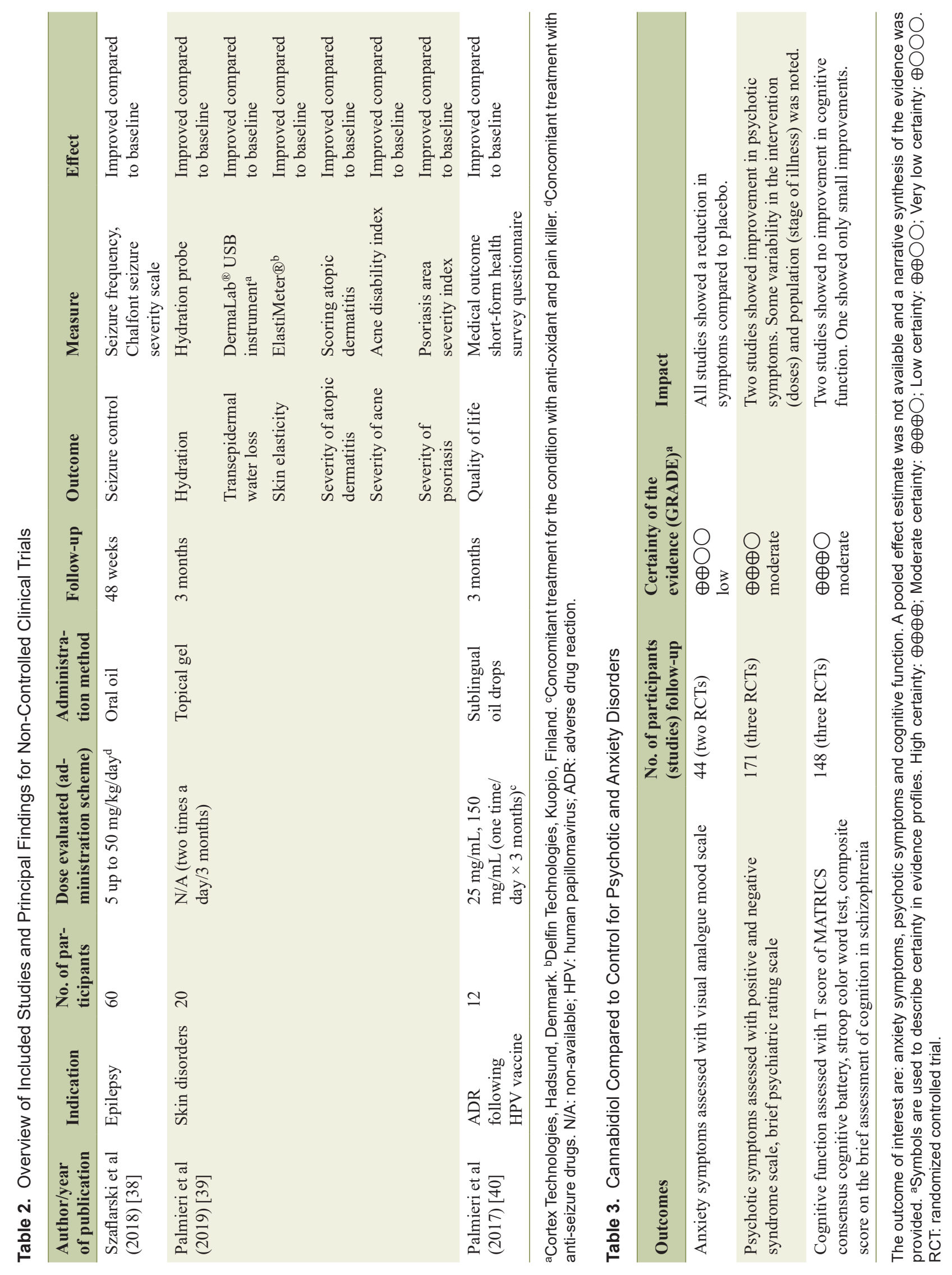


the studies was judged at low risk of bias, four at uncertain risk and four at high risk. Single doses of pure CBD 150 - $900 \mathrm{mg}$ in the form of gelatin capsules were administered in the majority of the cases. Two individually randomized parallel-group trials evaluated the effect of CBD on subjective anxiety on patients with SAD [17] or healthy subjects [18, 19] during simulated public speaking. These studies reported that acute oral administration of $600 \mathrm{mg}$ (SAD subjects) and $300 \mathrm{mg}$ (healthy subjects) of CBD reduced anxiety assessed by the visual analogue mood scale compared to placebo. Linares et al (2018) and Zuardi et al (2017) also tested other doses and observed no effect on anxiety levels after acute administration of 150, 600 and $900 \mathrm{mg}$ of CBD on anxiety levels. No effect was found for the physiological measures such as heart rate and systolic/ diastolic blood pressure [17-19]. Another small, individually randomized parallel-group trial, reported that acute administration of oral CBD $(600 \mathrm{mg})$ had a negative effect on anxiety levels, measured with the Beck's anxiety inventory, compared to placebo [20]. Single oral doses of 400 and $600 \mathrm{mg}$ decreased anxiety and increased mental sedation measured with visual analogue mood scale in two individually randomized crossover trials $[21,22]$. Two small non-RCTs administered 600 $\mathrm{mg}$ of oral CBD to health volunteers. One found no difference between placebo and CBD group on anxiety levels [23], while the other found a reduction on anxiety measured with visual analogue mood scale [24].

One randomized cross-over trial measured reactivity to negative stimulus through behavioral tasks and reported no effect at different incremental single doses of CBD (300, 600 and $900 \mathrm{mg}$ ). This measure was based on the hypotheses that drug anxiolytic effect should be manifested through changes in responses to negative stimuli, which also occur with single doses for common anxiolytics [25]. A cluster randomized cross-over trial assessed the effect of small doses $(16 \mathrm{mg})$ administered through inhalation on fear extinction and consolidation and found that it was effective when compared to placebo [26]. Das et al (2013) found that acute administration of $32 \mathrm{mg}$ of $\mathrm{CBD}$, before training, enhances extinction of conditioned fear responses [27].

\section{Psychosis}

Four randomized parallel-group trials (196 participants) assessed the role of CBD on cognitive impairment and psychotic symptoms in patients with psychotic disorders (schizophrenia) [28-31]. One study was judged as being at high risk of bias and three at uncertain risk of bias. Studies included patients with a confirmed diagnosis of schizophrenia. CBD was administered orally, in the form of oral gelatin capsules with doses ranging from 600 to $1,000 \mathrm{mg} /$ day and the effects were compared to a placebo and active control. Boggs et al (2018) assessed the effects of CBD (600 mg/day for 6 weeks) as an adjunctive treatment in chronic schizophrenia patients, for a period of 6 weeks. No significant difference was observed between placebo and CBD group on cognitive function and psychotic symptoms [28]. Leweke et al (2012) included a small group of acute schizophrenia patients who were administered 200 up to $800 \mathrm{mg} /$ day for 4 weeks or amisulpride, a potent antip- sychotic. CBD was as effective as amisulpride in improving psychotic symptoms and associated with marked tolerability and safety, when compared with amisulpride [29]. McGurie et al (2019) administered 1,000 mg/day for 6 weeks to a group of schizophrenia patients as an adjunct to current antipsychotic treatment and found a significant improvement on positive psychotic symptoms and clinicians' impressions of illness improvement. Despite improvement on cognitive function and overall level of functioning, no significant difference was found compared to placebo [30]. Hallak et al (2010) included a small group of heterogeneous schizophrenia patients and administered a single dose of CBD (300 or $600 \mathrm{mg}$ ). They found no effect of CBD on selective attention, measured by Stroop color and word test [31].

\section{Substance use disorder}

In a randomized cross-over design trial, Haney et al (2016) tested a range $(200,400$ and $800 \mathrm{mg}$ ) of oral single doses of pure $\mathrm{CBD}$, on cannabis smokers to assess the reinforcing subjective and psychological effects of smoked cannabis. The authors found no evidence with this treatment and dose scheme, and CBD can reduce the reinforcing or positive effects of smoked cannabis in current smokers [32]. Optional use of inhaled pure CBD (400 $\mu \mathrm{g} /$ dose $)$ over 1 week produced positive effects with regard to nicotine addiction, as measured by a reduction on the number of cigarette smoked in a group of healthy smokers willing to quit the habit. However, CBD did not show effect on carving symptoms [33]. After overnight tobacco abstinence single doses of $800 \mathrm{mg}$ of CBD reduced the salience and pleasantness of cigarette cues which indicate that CBD may have a potential effect on motivational aspects of addiction [34]. Two studies were judged at uncertain risk of bias and one at high risk.

\section{Other conditions}

Three RCTs, all individually randomized parallel group, reported on different medical conditions (one study for each category) [28-37]. Doses ranged from 20 to $250 \mathrm{mg}$ /day with treatment duration from 8 to 13 weeks. Isolated compound or plant exacts rich in CBD were administered in the form of hard oral capsules or oil. One study was judged at uncertain risk of bias and two at high risk.

In one trial, $200 \mathrm{mg} /$ day was administered for 13 weeks as an adjunct to current treatment to patients with type 2 diabetes. Compared to placebo, CBD did not have a significant effect on the level of high-density lipoproteins, the primary endpoint for this study [35]. The effect of oral administration of CBD (20 $\mathrm{mg}$ /day for 8 weeks) on disease activity assessed by the Crohn's disease activity index was evaluated in a small group of patients with long-standing Crohn's disease taking concomitant medications. CBD had no effect on disease activity at the end of treatment and at 2 weeks follow-up [36]. Irvin et al (2018) reported no effects of CBD-rich extracts (100 mg/day up to 250 /day for 8 weeks) added to current treatment and ad- 
ministrated in the form of oral capsules to patients diagnosed with ulcerative colitis [37].

\section{Non-controlled intervention studies}

A label single arm trial [38] assessed long-term use of isolated CBD $(5 \mathrm{mg} / \mathrm{kg} /$ day incremental doses in some patients reaching $2,000 \mathrm{mg} /$ day) as an adjunct to anti-epileptic drugs, in patients refractory to conventional anti-seizure drugs and reported a decrease in seizure frequency (144.4 to 52.2, P = 0.01 ) and severity measured with the Chalfont seizure severity scale (from 80.7 at baseline to $39.2, \mathrm{P}<0.0001$ ) at week 12 , with values being stable thereafter (total follow-up 48 weeks). The other two studies $[39,40]$ included reported on the chronic administration effect of CBD in the form of a topical cream for skin disorders and sublingual oil drops $(25 \mathrm{mg} /$ day up to $150 \mathrm{mg} /$ day for 12 weeks) for adverse drugs reaction (ADR) following human papilloma virus (HPV) vaccine, with a 3-month follow-up period each. The topical application produced positive results for serous skin inflammatory conditions such a psoriasis, acne and related scars, with no side effects being reported [39]. The sublingual administration of CBD also improved the quality of life in girls presenting with ADR following HPV vaccine when added to standard treatment. The evidence however arises from two case series and this should be taken into consideration when considering the results from this study [40].

\section{AEs}

AEs were reported in 10 studies. In most studies reporting on AEs [20, 28-30, 35-38, 40, 41], CBD was administered chronically, with follow-up ranging from 6 to 48 weeks, except for two studies where AEs were reported after a single dose of CBD (600 and $750 \mathrm{mg}$, respectively) [20-41]. The total number of reported patients, withdrawing from the study due to experience of side effects, was 16 in the CBD group. One study reported that side effects did not differ between the CBD and placebo groups after 8 weeks of follow-up [36], and another study reported a decrease of AEs 12 weeks after CBD initiation [38]. In another study side effects were monitored by answering question to a questionnaire [37]. The only study comprising $\mathrm{CBD}$ effects with an active group receiving an antipsychotic drug, amisulpride, in patients with schizophrenia reported that the CBD group had fewer extrapyramidal symptoms, less weight gain and prolactin release [29]. Results for the remaining studies, by indication and follow-up, for the number of participants experiencing any AEs compared to placebo AEs, classified by primary system organ class are reported in Table 4.

\section{Discussion}

This systematic review assessed the dosage schemes, effects and safety issues associated with the use of CBD. We included

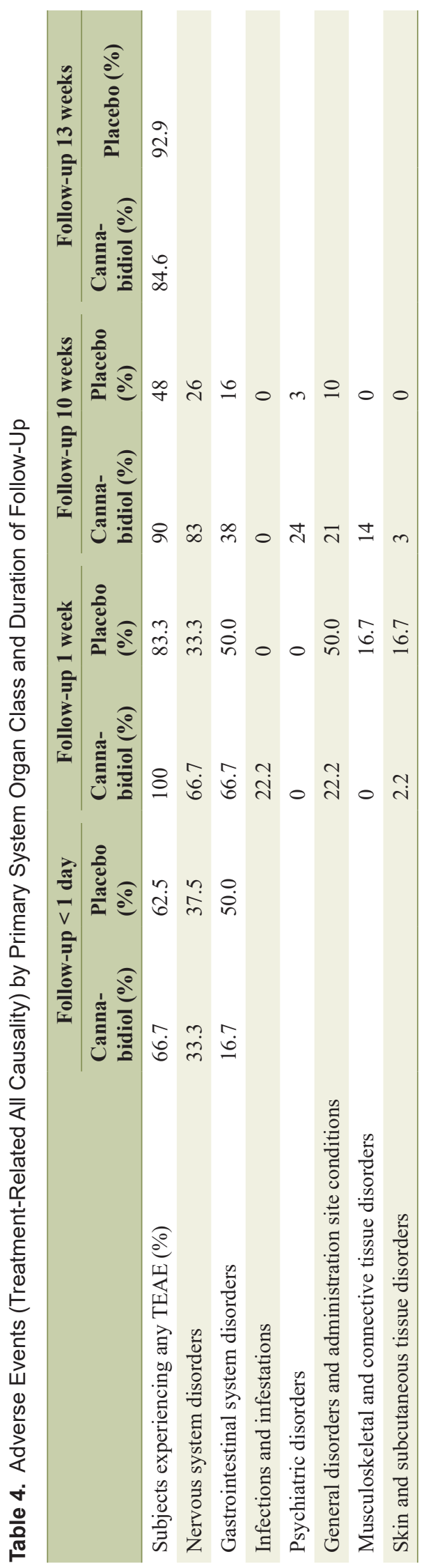


20 RCTs, two non-RCTs and three observational studies for all indications of CBD use. In summary, 20 RCTs evaluated efficacy of CBD-use for schizophrenia, anxiety, Crohn's disease, ulcerative colitis, dyslipidemia, nicotine addiction and cannabis use disorder. Most of these studies reported positive effect of CBD on anxiety, schizophrenia, tobacco addiction and minor effects or no effect on primary outcome measures for Crohn's disease, ulcerative colitis, dyslipidemia and cannabis use disorder. All observational studies (skin disorders, ADR following HPV vaccine and epilepsy) reported positive effect of CBD when compared to baseline measures.

Regarding safety issues, most studies reported no AEs with acute administration and mild to moderate adverse effects with chronic administration. In comparison to other drugs, a better side effect profile was presented $[29,30]$. The chronic administration of CBD for most of the included studies had a duration of few weeks ( 4 - 6 weeks). There is a need for longer term safety data and systematic/uniform reporting of AEs to better weight benefit and harms in future reviews. At least, two systematic reviews have been published on safety and side effects of CBD. The comprehensive review by Bergamaschi et al included animal and clinical studies reporting a favorable safety profile of CBD in humans [42]. The other review was an update of the previous focusing more on clinical data. The authors reported that most commonly side effects were tiredness, diarrhea and changes of appetite/weight. In comparison to other treatments used for epilepsy and psychotic disorders, CBD administration presented fewer side effects [43].

Most of the studies included in this review showed an improvement of anxiety levels after single doses of oral CBD with doses ranging from 300 to $600 \mathrm{mg}[17-19,21,22,24$, $26,27]$. It is important to note that only two studies included a clinical population (SAD) while the majority involved healthy subjects. Both studies tested acute administration of CBD and found a reduction in subjective anxiety. A systematic review on specific anxiety conditions which included data from clinical and pre-clinical studies found strong evidence arising from pre-clinical studies to support the anxiolytic effects of CBD, and similarly that evidence from human studies was limited to acute dosing in mostly healthy subjects [44]. There is a need for better designed studies to evaluate the therapeutic potential of CBD in this clinical population, possibly with chronic dosing in a relevant clinical population.

A systematic review providing evidence from clinical studies, mainly randomized clinical trial and case series, on the efficacy of CBD in the treatment of schizophrenia and/or substance abuse disorders observed large differences in study population, doses and administration [45]. In the present review, two principal outcomes were considered for schizophrenia patients, psychotic symptoms and cognitive functioning. CBD had positive effect on psychotic symptoms especially in acutely psychotic patients [29], while it had small or no effect on chronic schizophrenia patients who had been treated with anti-psychotics. The possibility that larger effects may be observed for patients in the early phases of the disease had been suggested [28, 45]. Regarding cognitive function little or no effects were observed after chronic or acute administration. A recent systematic review including 27 RTCs that investigated the effects of CBD on different psychiatric disorders such as psychosis, moods disorders and anxiety found that because of large heterogeneity across studies CBD doses, formulations and the study populations, it was not possible to make definitive conclusion about clinical effects [44]. The authors suggested that large-scale placebo controlled studies are needed to investigate the effects of $\mathrm{CBD}$ as an adjunct treatment for psychiatric disorder [46].

No effect was reported in the studies included in this review for the treatment of Crohn's disease, ulcerative colitis and type 2 diabetes with doses ranging between 20 and $250 \mathrm{mg}$ /day for a treatment duration of $8-13$ weeks [38-40]. While positive results were reported on seizure improvement with doses ranging from 350 to $2,000 \mathrm{mg} /$ day and on the quality of life for patients with ADRs following HPV vaccine, with doses ranging from 25 to $150 \mathrm{mg} /$ day, after 12 weeks of treatment [31, 33]. No definitive conclusion can be made on doses required for a positive effect since this may depend on the outcome assessed and study population.

In all studies, expect for one that used plant extracts [37], purified CBD was administered. Data derived from pre-clinical animal models indicate that purified CBD may have a bell shape response [47] which was also confirmed in two studies included in this review $[25,26]$. It is likely that the use of a single cannabinoid may be inferior to the extract where other components synergize with CBD to obtain the desired effect, known as the "entourage effect" [48]. Further, the main route of administration for the studies included in this review was oral (either in the form of capsules or sublingual oil). Animal studies suggest that oral bioavailability is low [48]; on the other hand, as highlighted in a recent systematic review on the pharmacokinetics of the CBD in humans [49], there is a lack of data in humans [50].

We used the Cochrane risk of bias tool to assess the included RCTs [8]. Overall, several methodological weaknesses were identified, e.g. selective outcome reporting, inadequate randomization and blinding. Further, sample sizes were very small in most studies significantly decreasing strength of detecting differences between study groups. An important finding of this review is the heterogeneous use of doses, dosage schemes and formulations (inhalation, oral capsules and sublingual oil, topical gel) across all indications of CBD. This has several implications. Besides excluding the option of pooling data for a metaanalysis to evaluate efficacy, the consequence of non-consensus of CBD dose is important when evaluating safety issues. Commercially, several online "dose-calculators" are available for dose recommendations (e.g. https://www.mydosage.com/); the data to support such calculators remain unclear on appropriate doses for efficacy although it seems reasonable to guide patients to safe dosage schemes and avoid adverse effects and gathering more data on how CBD is commonly being applied [51].

Although this review followed the recommendations for rigorous systematic reviews, it bears limitations here amongst a language and date restriction applied as well as a search strategy limited to electronic databases. However unlikely, other studies may not have been identified which can limit the applicability of the findings. Most of the disorders/diseases were only evaluated in single studies providing limited experience and no option to pool data, and some studies failed to present specific inclusion criteria meaning no restriction as to study group. The 
studies identified that evaluated identical conditions, regrettably employed different endpoints or tools of assessment.

RCTs are needed to confirm the effect of CBD on skin disorders, epilepsy and ADR following HPV vaccine. In addition, large and robust RTCs are needed to confirm the effects of CBD particularly on anxiety and psychosis. Studies should adhere to reporting standards for trials and use similar outcomes, standard measurements/tools to assess outcomes, and comparable treatment regimens to allow comparisons in future review studies. International guidelines should be implemented before the justification of further trials.

\section{Conclusions}

Studies included in this review evaluated mainly oral administration of purified CBD with placebo group as a comparator. However, there was larger heterogeneity between studies with regard to the population, schemes and doses of $\mathrm{CBD}$, outcomes and tool of measurement. There is some evidence, even though low quality, that supports anxiolytic effect of acute administration of oral CBD. There is moderate-quality evidence that chronic and acute administration of CBD can improve psychotic symptoms in schizophrenia patients. Further, large RCTs are needed to confirm the effect of CBD for the treatment of Crohn's disease, ulcerative colitis, dyslipidemia and cannabis use disorders. Insufficient data regarding safety issues were provided, but most studies reported no AEs with acute administration and mild to moderate adverse effects with chronic administration.

\section{Acknowledgments}

None to declare.

\section{Financial Disclosure}

Nordic Cannabis Research Institute (www.ncrinstitute.com).

\section{Conflict of Interest}

None to declare.

\section{Author Contributions}

CL designed the study. CL and JSH participated in data acquisition, extraction, analysis and drafted the final work. Authors reviewed the final version of the manuscript and approved it for publication.

\section{Data Availability}

The data supporting the findings of this study are available from the corresponding author upon request.

\section{Abbreviations}

2-AG: 2-arachidonoylglycerol; $\mathrm{CBD}$ : cannabidiol; $\mathrm{CB}_{1}$ : cannabinoid receptor type $1 ; \mathrm{CB}_{2}$ : cannabinoid receptor type 2 ; $\triangle 9$-THC: delta-9-tetrahydrocannabinol; FAAH: fatty acid amide hydrolase; GPR55: G protein-coupled receptor 55; IFN $\gamma$ : interferon gamma; IL: interleukin; LPS: lipopolysaccharide; PGE2: prostaglandin E2; NO: nitric oxide

\section{References}

1. Schubart CD, Sommer IE, van Gastel WA, Goetgebuer RL, Kahn RS, Boks MP. Cannabis with high cannabidiol content is associated with fewer psychotic experiences. Schizophr Res. 2011;130(1-3):216-221.

2. Pisanti S, Malfitano AM, Ciaglia E, Lamberti A, Ranieri R, Cuomo G, Abate M, et al. Cannabidiol: State of the art and new challenges for therapeutic applications. Pharmacol Ther. 2017;175:133-150.

3. Morales P, Hurst DP, Reggio PH. Molecular targets of the phytocannabinoids: a complex picture. Prog Chem Org Nat Prod. 2017;103:103-131.

4. Esposito G, Filippis DD, Cirillo C, Iuvone T, Capoccia E, Scuderi C, Steardo A, et al. Cannabidiol in inflammatory bowel diseases: a brief overview. Phytother Res. 2013;27(5):633-636.

5. Campos AC, Fogaca MV, Sonego AB, Guimaraes FS. Cannabidiol, neuroprotection and neuropsychiatric disorders. Pharmacol Res. 2016;112:119-127.

6. Boychuk DG, Goddard G, Mauro G, Orellana MF. The effectiveness of cannabinoids in the management of chronic nonmalignant neuropathic pain: a systematic review. J Oral Facial Pain Headache. 2015;29(1):7-14.

7. Devinsky O, Marsh E, Friedman D, Thiele E, Laux L, Sullivan J, Miller I, et al. Cannabidiol in patients with treatment-resistant epilepsy: an open-label interventional trial. Lancet Neurol. 2016;15(3):270-278.

8. Turner SE, Williams CM, Iversen L, Whalley BJ. Molecular pharmacology of phytocannabinoids. Prog Chem Org Nat Prod. 2017;103:61-101.

9. Cascio MG, Pertwee RG, Marini P. The pharmacology and therapeutic potential of plant cannabinoids. In: Cannabis Sativa L. Botany and Biotechnology. Springer International Publishing. 2017; p. 207-225.

10. McPartland JM, Glass M, Pertwee RG. Meta-analysis of cannabinoid ligand binding affinity and receptor distribution: interspecies differences. Br J Pharmacol. 2007;152(5):583-593.

11. Laprairie RB, Bagher AM, Kelly ME, Denovan-Wright EM. Cannabidiol is a negative allosteric modulator of the cannabinoid CB1 receptor. Br J Pharmacol. 2015;172(20):4790-4805.

12. Russo E, Guy GW. A tale of two cannabinoids: the therapeutic rationale for combining tetrahydrocannabinol and cannabidiol. Med Hypotheses. 2006;66(2):234-246.

13. De Filippis D, Esposito G, Cirillo C, Cipriano M, De Winter BY, Scuderi C, Sarnelli G, et al. Cannabidiol re- 
duces intestinal inflammation through the control of neuroimmune axis. PLoS One. 2011;6(12):e28159.

14. Izzo AA, Borrelli F, Capasso R, Di Marzo V, Mechoulam R. Non-psychotropic plant cannabinoids: new therapeutic opportunities from an ancient herb. Trends Pharmacol Sci. 2009;30(10):515-527.

15. Higgins JP, Altman DG, Gotzsche PC, Juni P, Moher D, Oxman AD, Savovic J, et al. The Cochrane Collaboration's tool for assessing risk of bias in randomised trials. BMJ. 2011;343:d5928.

16. Murad MH, Mustafa RA, Schunemann HJ, Sultan S, Santesso N. Rating the certainty in evidence in the absence of a single estimate of effect. Evid Based Med. 2017;22(3):85-87.

17. Bergamaschi MM, Queiroz RH, Chagas MH, de Oliveira DC, De Martinis BS, Kapczinski F, Quevedo J, et al. Cannabidiol reduces the anxiety induced by simulated public speaking in treatment-naive social phobia patients. Neuropsychopharmacology. 2011;36(6):1219-1226.

18. Linares IM, Zuardi AW, Pereira LC, Queiroz RH, Mechoulam R, Guimaraes FS, Crippa JA. Cannabidiol presents an inverted U-shaped dose-response curve in a simulated public speaking test. Braz J Psychiatry. 2019;41(1):9-14.

19. Zuardi AW, Rodrigues NP, Silva AL, Bernardo SA, Hallak JEC, Guimaraes FS, Crippa JAS. Inverted U-shaped dose-response curve of the anxiolytic effect of cannabidiol during public speaking in real life. Front Pharmacol. 2017;8:259.

20. Hundal H, Lister R, Evans N, Antley A, Englund A, Murray $\mathrm{RM}$, Freeman $\mathrm{D}$, et al. The effects of cannabidiol on persecutory ideation and anxiety in a high trait paranoid group. J Psychopharmacol. 2018;32(3):276-282.

21. Crippa JA, Zuardi AW, Garrido GE, Wichert-Ana L, Guarnieri R, Ferrari L, Azevedo-Marques PM, et al. Effects of cannabidiol (CBD) on regional cerebral blood flow. Neuropsychopharmacology. 2004;29(2):417-426.

22. Crippa JA, Derenusson GN, Ferrari TB, Wichert-Ana L, Duran FL, Martin-Santos R, Simoes MV, et al. Neural basis of anxiolytic effects of cannabidiol (CBD) in generalized social anxiety disorder: a preliminary report. J Psychopharmacol. 2011;25(1):121-130.

23. Martin-Santos R, Crippa JA, Batalla A, Bhattacharyya S, Atakan Z, Borgwardt S, Allen P, et al. Acute effects of a single, oral dose of d9-tetrahydrocannabinol (THC) and cannabidiol (CBD) administration in healthy volunteers. Curr Pharm Des. 2012;18(32):4966-4979.

24. Bhattacharyya S, Morrison PD, Fusar-Poli P, MartinSantos R, Borgwardt S, Winton-Brown T, Nosarti C, et al. Opposite effects of delta-9-tetrahydrocannabinol and cannabidiol on human brain function and psychopathology. Neuropsychopharmacology. 2010;35(3):764-774.

25. Arndt DL, de Wit H. Cannabidiol does not dampen responses to emotional stimuli in healthy adults. Cannabis Cannabinoid Res. 2017;2(1):105-113.

26. Hindocha C, Freeman TP, Schafer G, Gardener C, Das RK, Morgan CJ, Curran HV. Acute effects of delta-9-tetrahydrocannabinol, cannabidiol and their combination on facial emotion recognition: a randomised, double-blind, placebo-controlled study in cannabis users. Eur Neu- ropsychopharmacol. 2015;25(3):325-334.

27. Das RK, Kamboj SK, Ramadas M, Yogan K, Gupta V, Redman E, Curran HV, et al. Cannabidiol enhances consolidation of explicit fear extinction in humans. Psychopharmacology (Berl). 2013;226(4):781-792.

28. Boggs DL, Surti T, Gupta A, Gupta S, Niciu M, Pittman B, Schnakenberg Martin AM, et al. The effects of cannabidiol (CBD) on cognition and symptoms in outpatients with chronic schizophrenia a randomized placebo controlled trial. Psychopharmacology (Berl). 2018;235(7):19231932.

29. Leweke FM, Piomelli D, Pahlisch F, Muhl D, Gerth CW, Hoyer C, Klosterkotter J, et al. Cannabidiol enhances anandamide signaling and alleviates psychotic symptoms of schizophrenia. Transl Psychiatry. 2012;2:e94.

30. McGuire P, Robson P, Cubala WJ, Vasile D, Morrison PD, Barron R, Taylor A, et al. Cannabidiol (CBD) as an adjunctive therapy in schizophrenia: a multicenter randomized controlled trial. Am J Psychiatry. 2018;175(3):225-231.

31. Hallak JE, Machado-de-Sousa JP, Crippa JA, Sanches RF, Trzesniak C, Chaves C, Bernardo SA, et al. Performance of schizophrenic patients in the Stroop Color Word Test and electrodermal responsiveness after acute administration of cannabidiol (CBD). Braz J Psychiatry. 2010;32(1):56-61.

32. Haney M, Malcolm RJ, Babalonis S, Nuzzo PA, Cooper ZD, Bedi G, Gray KM, et al. Oral Cannabidiol does not Alter the Subjective, Reinforcing or Cardiovascular Effects of Smoked Cannabis. Neuropsychopharmacology. 2016;41(8):1974-1982.

33. Morgan CJ, Das RK, Joye A, Curran HV, Kamboj SK. Cannabidiol reduces cigarette consumption in tobacco smokers: preliminary findings. Addict Behav. 2013;38(9):2433-2436.

34. Hindocha C, Freeman TP, Grabski M, Stroud JB, Crudgington $\mathrm{H}$, Davies AC, Das RK, et al. Cannabidiol reverses attentional bias to cigarette cues in a human experimental model of tobacco withdrawal. Addiction. 2018;113(9):1696-1705.

35. Jadoon KA, Ratcliffe SH, Barrett DA, Thomas EL, Stott C, Bell JD, O'Sullivan SE, et al. Efficacy and safety of cannabidiol and tetrahydrocannabivarin on glycemic and lipid parameters in patients with type 2 diabetes: a randomized, double-blind, placebo-controlled, parallel group pilot study. Diabetes Care. 2016;39(10):1777-1786.

36. Naftali T, Mechulam R, Marii A, Gabay G, Stein A, Bronshtain M, Laish I, et al. low-dose cannabidiol is safe but not effective in the treatment for crohn's disease, a randomized controlled trial. Dig Dis Sci. 2017;62(6):16151620.

37. Irving PM, Iqbal T, Nwokolo C, Subramanian S, Bloom S, Prasad N, Hart A, et al. A randomized, double-blind, placebo-controlled, parallel-group, pilot study of cannabidiolrich botanical extract in the symptomatic treatment of ulcerative colitis. Inflamm Bowel Dis. 2018;24(4):714-724.

38. Szaflarski JP, Bebin EM, Cutter G, DeWolfe J, Dure LS, Gaston TE, Kankirawatana P, et al. Cannabidiol improves frequency and severity of seizures and reduces adverse events in an open-label add-on prospective study. Epi- 
lepsy Behav. 2018;87:131-136.

39. Palmieri B, Laurino C, Vadala M. A therapeutic effect of cbd-enriched ointment in inflammatory skin diseases and cutaneous scars. Clin Ter. 2019;170(2):e93-e99.

40. Palmieri B, Laurino C, Vadala M. Short-Term Efficacy of CBD-Enriched Hemp Oil in Girls with Dysautonomic Syndrome after Human Papillomavirus Vaccination. Isr Med Assoc J. 2017;19(2):79-84.

41. Taylor L, Gidal B, Blakey G, Tayo B, Morrison G. A phase I, randomized, double-blind, placebo-controlled, single ascending dose, multiple dose, and food effect trial of the safety, tolerability and pharmacokinetics of highly purified cannabidiol in healthy subjects. CNS Drugs. 2018;32(11):1053-1067.

42. Bergamaschi MM, Queiroz RH, Zuardi AW, Crippa JA. Safety and side effects of cannabidiol, a Cannabis sativa constituent. Curr Drug Saf. 2011;6(4):237-249.

43. Iffland K, Grotenhermen F. An Update on Safety and Side Effects of Cannabidiol: A Review of Clinical Data and Relevant Animal Studies. Cannabis Cannabinoid Res. 2017;2(1):139-154.

44. Blessing EM, Steenkamp MM, Manzanares J, Marmar CR. Cannabidiol as a Potential Treatment for Anxiety
Disorders. Neurotherapeutics. 2015;12(4):825-836.

45. Batalla A, Janssen H, Gangadin SS, Bossong MG. The potential of cannabidiol as a treatment for psychosis and addiction: who benefits most? A systematic review. J Clin Med. 2019;8(7):1058.

46. Bonaccorso S, Ricciardi A, Zangani C, Chiappini S, Schifano F. Cannabidiol (CBD) use in psychiatric disorders: A systematic review. Neurotoxicology. 2019;74:282-298.

47. Gallily R, Yekhtin Z, Hanus LO. Overcoming the bell shaped dose response of cannabidiol by using cannabis extract enriched in cannabidiol. Pharmacology \& Pharmacy. 2015;6(2):75-85.

48. Russo EB. Taming THC: potential cannabis synergy and phytocannabinoid-terpenoid entourage effects. Br J Pharmacol. 2011;163(7):1344-1364.

49. Millar SA, Stone NL, Yates AS, O'Sullivan SE. A Systematic Review on the Pharmacokinetics of Cannabidiol in Humans. Front Pharmacol. 2018;9:1365.

50. Millar SA, Stone NL, Bellman ZD, Yates AS, England TJ, O'Sullivan SE. A systematic review of cannabidiol dosing in clinical populations. Br J Clin Pharmacol. 2019;85(9):1888-1900.

51. https://www.mydosage.com. 